\title{
Asymptomatic Pediatric Covid-19 Presenting with Thrombocytopenia - A Rare Finding
}

\author{
Deepali Ambike ${ }^{1}$ (D) $\cdot$ Komal Bijarniya $^{1}$
}

Received: 25 June 2020 / Accepted: 21 August 2020 / Published online: 28 August 2020

(C) Dr. K C Chaudhuri Foundation 2020

To the Editor: Severe Acute Respiratory Syndrome Corona Virus 2 (SARS-CoV-2), a new novel corona virus is the cause for the present pandemic with significant morbidity and mortality. Clinical manifestations are usually mild among children. Since many children can be asymptomatic carriers, it is essential to identify and isolate them for reducing the transmission [1].

We report two pediatric SARS-CoV-2 cases diagnosed positive by RT-PCR admitted in our hospital which is dedicated for Covid and is under local corporation body in Pune. The RT-PCR was done at National Institute of Virology which does not give $\mathrm{Ct}$ values as they do the tests on a large scale for all government hospitals in Pune and outside Pune.

Both cases were totally asymptomatic on presentation, admitted as a high risk contacts; both parents were positive for SARS-CoV-2 on RT-PCR test. Standard Covid-19 treatment protocol was initiated as per Ministry of Health and Family Welfare (MOHFW) guidelines and AIIMS protocol. First case was an asymptomatic 5-y-old male child who came positive after $3 \mathrm{~d}$ of exposure to his parents and laboratory findings of normal $\mathrm{Hb}, \mathrm{WBC}-7070$ /cumm, N - 38.6\%, L - 52.4\%, E $-2.9 \%, \mathrm{M}-4.6 \%$ with platelet count $-30,800 / \mathrm{mcL}$ [Grade 3 thrombocytopenia (As per NCI grading) on Day 1 of admission]. The platelet count slowly increased by day 10 of treatment to $90,000 / \mathrm{mcL}$. Case 2 was a 4 -y-old female child from another family who came positive after $2 \mathrm{~d}$ of exposure to positive parents; totally asymptomatic clinically. Lab parameters on Day 1 of admission showed normal $\mathrm{Hb}$, WBC - 4360/ cumm, N-24\%, L- 63\%, E- 6\%, M-2.3\% and platelet count $-66,900 / \mathrm{mcL}$ (Grade 2 thrombocytopenia) which by Day 4 of admission increased to $1,45,000 / \mathrm{mcL}$. The NL ratio (NLR) of

Deepali Ambike

ambikedeepa@gmail.com

1 Department of Pediatrics, PCMC, S Postgraduate Institute \& Yashwantrao Chawan Memorial Hospital, Pune 18, Pimpri, Maharashtra, India case 1 was high on admission- 0.8 and decreased to 0.4 on discharge. Case 2 had 0.4 NL ratio on admission and was same on discharge. Both the patients recovered by Day 14 and were discharged after their RT-PCR was negative for SARS-CoV-2. Neither of them showed any petechaie or purpura nor any bleeding manifestations. Both tested negative for Dengue NS1 test. However, other causes of thrombocytopenia could not be ruled out which is a limitation of this paper. We have successfully treated and discharged 175 pediatric patients of SARS-CoV- 2, all were asymptomatic, but only two of them had these lab derangements.

In a case series by Hu et al. of 24 asymptomatic infections, four $(16.7 \%)$ cases had lymphopenia and other four had leukopenia but none of them had thrombocytopenia [2]. Review article by Ludvigsson has stated lymphopenia as a rare finding [3]. Zimmermann et al. noted that the white blood cell count is typically normal or reduced with decreased neutrophil and/or lymphocyte counts and thrombocytopenia may occur [4]. We also demonstrated a higher NLR in both cases which could be predictive of on-going inflammation. Although majority of the Pediatric Covid-19 cases are asymptomatic or mildly symptomatic, in incidentally detected thrombocytopenia, no intervention is needed apart from follow-up of the counts and their NL ratio also needs to be monitored and can be a prognostic predictor before deciding the discharge.

Acknowledgements National Institute of Virology for the test reports and permission taken for publication.

\section{Compliance with Ethical Standards}

Conflict of Interest None.

\section{References}

1. Wu YC, Chen CS, Chan YJ. The outbreak of COVID-19: an overview. J Chin Med Assoc. 2020;83:217-20. 
2. Hu Z, Song C, Xu C, et al. Clinical characteristics of 24 asymptomatic infections with COVID-19 screened among close contacts in Nanjing. China Sci China Life Sci. 2020;63:706-11.

3. Jonas F. Ludvigsson: systematic review of COVID-19 in children shows milder cases and a better prognosis than adults. Acta Paediatrica. 2020;109:1088-95.

4. Zimmermann P, Curtis N. Coronavirus infections in children including COVID-19 an overview of the epidemiology, clinical features, diagnosis, treatment and prevention options in children. Pediatr Infect Dis J. 2020;39:355-68.

Publisher's Note Springer Nature remains neutral with regard to jurisdictional claims in published maps and institutional affiliations. 